\title{
MS02-P01 | STRUCTURE-BASED DRUG DESIGN TO TACKLE DISORDERS OF HAEM
}

\section{BIOSYNTHESIS}

Arruda Bezerra, Gustavo (University of Oxford - Structural Genomics Consortium, Oxford, GBR); Bailey, Henry (University of Oxford, Oxford, GBR); Foster, William (University of Oxford, Oxford, GBR); Rembeza, Elzbieta (University of Oxford, Oxford, GBR); Yue, Wyatt (University of Oxford, Oxford, GBR)

X-linked protoporphyria (XLP) is caused by gain-of-function mutations in erythroid-specific aminolevulinate synthase (ALAS2), the first and rate-limiting enzyme in haem biosynthesis. Additionally, inherited defects downstream of ALAS2 in the haem biosynthesis pathway of erythroids lead to several porphyrias. In this work, we aim to exploit inhibition of ALAS2 as substrate reduction therapy, whereby decreasing the influx of toxic haem intermediates in the pathway would ameliorate the aforementioned disorders. We have established a structural biology pipeline to generate milligram quantities of recombinant human ALAS2, suitable for large-scale crystallisation experiments. Hundreds of ALAS2 crystals were each soaked with a chemical fragment from a custom library, and their respective co-crystal structures were solved, in a week's timeframe, to identify fragments bound to ALAS2 as starting points for inhibitor design. From solving 295 crystal structures of ALAS2, we identified 19 bound fragments clustered in three different regions of the ALAS2 dimer interface, close to the C-terminal extension that is unique among higher eukaryotes. We have developed biophysical assays to evaluate the binding of fragments to ALAS2 and their impacts on enzyme activity, guiding us in future cycles of medicinal chemistry and drug design.As an obligate dimer, the ALAS2 active site is formed by residues from two subunits. This feature provides opportunities to develop inhibitors from fragments located at the dimer interface, aimed at disrupting protein dimerization. Additionally, fragments interacting with the mobile C-terminal extension, a key region for catalysis, could be developed into inhibitors aimed at restricting substrate access to the active site. 